\title{
1986 MRS Show Exhibitors
}

\author{
(as of October 8, 1986) \\ \# Indicates Exhibitors Booth Numbers
}

Academic Press - \#920

Sea Harbor Drive

Orlando, FL 32887

Contact: Betsy Carter

AG Associales - \#607

1325 Borregas Avenue

Sunnyvale, CA 94089

Contact: Deborah McGowan

American Institute of Physics - \#808, 809

335 East 45th Street

New York, NY 10017

Contact: Mark Dazzo

American Instruments, Inc. - \#105

186 Port Reading Avenue

Port Reading, NJ 07064

Contact: Robert Schwartz

Amplifier Research - \#206

160 Schoolhouse Road

Souderton, PA 18964

Contact: Dan Roth

Anatech, Ltd. - $\# 603,604$

5510 Vine Street

Alexandria, VA 22310

Contact: George Barr

Atomika Technische Physic GmbH - \#107

Postfach 450135

D-8000 Munich 45

Federal Republic of Germany

Contact: Dr. J.C. Maul

Bio-Rad Semiconductor Measurement

Systems - \#910

237 Putnam Avenue

Cambridge, MA 02139

Contact: Gerhard Kneissel

Blake Industries - \#703, 704

660 Jerusalem Road

Scotch Plains, NJ 07076

Contact: David C. Rognlie

Brimrose Corporation of America - $\$ 207$

7720 Belair Road

Baltimore, MD 21236

Contact: Ravi Ananth

Edmund Buhler Co. - \#935

P.O. Box 1021

Malibu, CA 90265

Contact: Bill Smith

Callery Chemical Co. $-\# 933$

P.O. Box 429

Pittsburgh, PA 15230

Contact: Samuel A. McCalmont

Cameca Instruments, Inc. - \#707

2001 W. Main Street

Stamford, CT 06902

Contact: Thomas Fisher

Ceramaseal - \#610

P.O. Box 25

New Lebanon Center, NY 12126

Contact: Gene Sawin

Commonwealth Scientific Corporation - \#608

500 Pendleton Street

Alexandria, VA 22314

Contact: Steven L. Collins

Cryosystems - \#928, 929

1802 W. Grant Road, Suite 122

Tucson, AZ 85745

Contact: Michael Swank
Denton Vacuum, Inc. - \#401

2 Pinoak Avenue

Cherry Hill, NJ 08003

Contact: Robert Specht

Ealing Electro-Optics, Inc. - \#112

22 Pleasant Street

South Natick, MA 01769

Contact: Mary L. Amiot

Eaton Corporation - $\# 301,302,303$

4001 N. First

San Jose, CA 95134

Contact: Judy Ruehle

Edwards High Vacuum - \#926

3279 Grand Island Blvd.

Grand Island, NY 14072

Contact: Rich Miecyjak

EG\&G Princelon Applied Research - \$940

6 DeAngelo Drive

Bedford, MA 01730

Contact: Lou Rubin

Elsevier Science Publishing - \#917

52 Vanderbilt Avenue

New York, NY 10017

Contact: Randy Schneiderman

Emcore - \#308

111 Corporate Blvd.

S. Plainfield, NJ 07080

Contact: Dr. Leslie G. Polgar

Enraf-Nonius - \#709

390 Central Avenue

Bohemia, NY 11716

Contact: Graheme J.B. Williams, PhD

Charles Evans \& Associates - \#810, 811

301 Chesapeake Drive

Redwood City, CA 94063

Contact: Donald H. Wayne

Gaertner Scientific Corporation - \#201

1201 Wrightwood Avenue

Chicago, IL 60614

Contact: Rusty Kutko

Gatan - \#111

780 Commonwealth Drive

Warrendale, PA 15086

Contact: Kevin Scudder

General lonex Corporation - \#402, 403, 404

19 Graf Road

Newburyport, MA 01950

Contact: David Stern

Granville-Phillips - \#609

5675 E. Arapahoe Avenue

Boulder, CO 80303

Contact: Kathy L. Valliere

High Voltage Engineering Europa BV - \#106

P.O. Box 99

3800 AB Amersfoort

The Netherlands

Contact: R. Goethals

HPS Corporation - \#925

5330 Sterling Drive

Boulder, CO 80301

Contact: Karen Smith

Huntington Laboratories -

1040 L'avenida

Mountain View, CA 94043

Contact: Doug Marcum
Ilford Ltd. - \#208

Mobberley, Knutsford

Cheshire, UK WA16 6HA

Contact: Jane Johnson

Industrial Equipment \& Sales Corporation \#108, 109

36 Washington Street

Wellesley, MA 02146

Contact: Ned Dewey

Innovative Technology, Inc. - \#309

205 Willow Street

South Hamilton, MA 01982

Contact: Peter M. Calandra

Instruments S.A./Riber - \#501, 502

173 Essex Avenue

Metuchen, NJ 08840

Contact: Kristine B. Richter

Janis Research Co. - \#701

2 Jewel Drive

P.O. Box 696

Wilmington, MA 01887

Contact: Munir N. Jirmanus

JCPDS-International Centre for

Diffraction Data - \#914

1601 Park Lane

Swarthmore, PA 19081

Contact: James $\mathrm{H}$. Lang

JEOL USA, Inc. - \#601

11 Dearborn Road

Peabody, MA 01960

Contact: Jeanne C. Wilson

Journal of Materials Research - \#936

9800 McKnight Road, Suite 327

Pittsburgh, PA 15237

Contact: Gail Oare

Keithley Instruments, Inc./Instruments Div. \#922, 921

28775 Aurora Road

Cleveland, $\mathrm{OH} 44139$

Contact: Mary Jones

Kevex Corporation - \#708

1101 Chess Drive

Foster City, CA 94404

Contact: Helen Barke

Kimball Physics, Inc. - \#605, 606

Kimball Hill Road

Wilton, NH 03086-9742

Contact: Faye Bigarel

Lake Shore Cryotronics - \#911

64 East Walnut Street

Westerville, $\mathrm{OH} 43081$

Contact: David L. Cavelage

Lambda Physik - \#939

289 Great Road

Acton, MA 01720

Contact: Jack Andrellos

Lehighton Electronics - \#104

P.O. Box 328

Lehighton, PA 18235

Contact: Austin Blew

Leitz-IMS Co. $-\# 937$

900 Middlesex Tpk.

Building \#8

Billerica, MA 01821

Contact: David J. Elliott 
Kurt J. Lesker Co. - \#405

1515 Worthington Road

Clairton, PA 15025

Contact: Laurie Englert

Leybold-Heraeus Vacuum Products \#304, 305

5700 Mellon Road

Export, PA 15632

Contact: Eugene Fuselier

Materials by Metron - \#916

P O. Box 147

Allamuchy, NJ 07820

Contact: Melvin Hollander

Microscience, Inc. - \#902, 903

Forbès Business Center

182 Forbes Road

Braintree, MA 02184

Contact: Tricia Heirty

MKS Instruments, Inc. - \#806

34 Third Avenue

Burlington, MA 01803

Contact: Mike Thomas

Nanometrics - \#927

690 East Arques Avenue

Sunnyvale, CA 04086

Contact: John Scott

National Bureau of Standards - \#202, 203

Chemistry Building, Rm. B-311

Gaithersburg, MD 20899

Contact: Lee Klein

National Electrostatics Corporation - \#702

P.O. Box 310

Graber Road

Middleton, WI 53562

Contact: Gregory Norton

Neslab Instruments - \#710

P.O. Box 1178

Portsmouth, NJ 03801

Contact: Mark Zarembo

Neizsch Incörporated - \#310

119 Pickering Way

Exton, PA 19341

Contact: John E. Kelly III

Nicolet Analytical Instruments - \#905

5225 Verona Road

Box 4508

Madisön, WI 53711

Contact: Cheryl Bowen

NGS Associates - \#409

110 'Shawmut Road

Canton, MA 02021

Contact: Marie Kapsalis

North Eastern Analytical - \#509

17 Sherman Road

P.O. Box 25

Millis, PA 02054

Contact: Thomas J. Flanagan

NSA/Hitachi Scientific Instruments \#204, 205

460 E. Middlefield Road

Mountain View, CA 94043

Contact: Donna Armanino

Oriel Corporation - \#112

250 Long Beach Blvd.

P.O: Box 872

Stratford, CT 06497

Contact: Thomas Connelly

Oxford Instruments N.A. Inc. - \#802

3A Altred Circle

Bedford. MA 01730

Contact: Michael Cassidy

Perkin-Elmer - \#919

6509 Flying Cloud Drive

Eden Prairie, MN 55344

Contact: Lori Broecker
Philips Electronic Instruments - \#306, 307

85 McKee Drive

Mahwah, NJ 07430

Contact: Michele Martin

Photon Technology International - \#932

601 Ewing Street C-2

Princeton, NJ 08540

Contact: James D. Bleakley

Physicon Corporation - \#913

$221 \mathrm{Mt}$. Auburn Street

Cambridge, MA 02138

Contact: $H$. von Zweck

Physitec Corporation - \#410

206 Main Street

Norfolk, MA 02056

Contact: Peter Andreas

Plenum Publishing Corporation - \$923

233 Spring Street

New York, NY 10013

Contact: Eleonore Jentsch

Polymer Laboratories, Inc. - \#909

160 Old Farm Road

Amherst, MA 01002

Contact: Debra Bennett

Princeton Gamma-Tech, Inc. - \#503, 504

1200 State Road

Princeton, NJ 08540

Contact: Robert Nicastro

Process Products, Inc. - \#918

37 Flagship Drive

N. Andover, MA 01845

Contact: Phil Sampson

Questek, Inc. - \#209

44 Manning Road

Billerica, MA 01821

Contact: James Campbell

Rigaku/USA, Inc. - \#505, 506

3 Electronics Avenue

Danvers, MA 01923

Contact: Janet L. Anderson

Rudolph Research - $\$ 807$

One Rudolph Road

P O Box 1000

Flanders, NJ 07836

Contact: Richard L. Budzinski

Scintag, Inc. - \#924

3350 Scott Blvd.

Santa Clara, CA 95054

Contact: Elisabeth Rippstein

The Semi Group, Inc. - \#407, 408

107 San Zeno Way

Sunnyvale, CA 94086

Contact: James L. Corbin

Semiconductor Processing Co. - \#906

409 East First Street

Boston, MA 02127

Contact: Richard Sawyer

Siemens Corporate Research \& Support, Inc. - \#907, 908

186 Wood Avenue South

Iselin, NJ 08830

Contact: Doreen Reich

SOHIO Engineered Materials - \#210

Carborundum Center

P.O. Box 156

Niagara Falls, NY 14302

Contact: Rosa Capobianco

South Bay Technology, Inc. - \#510

5209 Tyler Avenue

Temple City. CA 91780

Contact: R.E. Henricks
Spectra Gases, Inc. - \#934

277 Coit Street

Irvington, NJ 07111

Contact: Valerie Reicheg

Spectramass, Inc. - \#803

P.O. Box 95034

Pittsburgh, PA 15223

Contact: Clyde Madsen

Spex Industries, Inc. - \#915

3880 Park Avenue

Edison, NJ 08820

Contact: John Noonan

Spire Corporation - \#406

Patriots Park

Bedford, MA 01730

Contact: Jeri Freedman

Springer-Verlag New York Publishing - \#904

175 Fifth Avenue

New York, NY 10010

Contact: Marsha J. Levell-Braggs

Stanford Research Systems - \#938

460 California Avenue

Palo Alto, CA 94306

Contact: John Pease

Structure Probe/SPI Supplies - \#805

535 East Gay Street

P.O. Box 656

West Chester, PA 19381-0656

Contact: Charles A. Garber, PhD

Surface Alloys Corporation - \#602

33 Cherry Hill Drive

Danvers, MA 01923

Contact: A.J. Armini

Surface Science Laboratories - \#110

1200 Charleston Road

Mountain View, CA 94043

Contact: Betsy Springer Smullen

Thermionics Laboratory, Inc. - \#804

P.O. Box 3711

22815 Sutro Street

Hayward, CA 94541

Contact: Mark Trujilio

Tracor Northern - \#705, 707

2552 W. Beltline Hwy.

Middleton, WI 53562

Contact: Joseph A. Mullen

UHV Instruments - \#930

1951 Hamburg Turnpike

Office Box 12

Buffalo, NY 14218

Contact: J.S. Vanderwoude

Universal Energy Systems - \#931

4401 Dayton-Xenia Road

Dayton, $\mathrm{OH} 45432$

Contact: Marty Barker

Varian - \#500

611 Hansen Way

Palo Alto, CA 94303

Contact: Mrs. Nathalie F. Gross

Varian Associates/Vacuum Products Division - \#400

121 Hartwell Avenue

Lexington, MA 02173

Contact: Brooke V. Thorley

VCR Group, Inc. - \#103

650 Fifth St. Suite 305

San Francisco, CA 94107

Contact: Ron Douglass

VG Instruments, Inc. - \#507, 508

300 Broad Street

Stamford, CT 06901

Contact: W. Allen Wolstenholme 